\title{
Top-down influences mediate hand bias in spatial attention
}

\author{
John P. Garza - Michael J. Strom • Charles E. Wright • \\ Ralph J. Roberts Jr. • Catherine L. Reed
}

Published online: 31 May 2013

(C) Psychonomic Society, Inc. 2013

\begin{abstract}
Spatial attention can be biased to locations near the hand. Some studies have found facilitated processing of targets appearing within hand-grasping space. In this study, we investigated how changing top-down task priorities alters hand bias during visual processing. In Experiment 1, we used a covert orienting paradigm with nonpredictive cues and emphasized the location of the hand relative to the target. Hands or visual anchors (boards) were placed next to potential target locations, and responses were made with the contralateral hand. Results indicated a hand-specific processing bias: Hand location, but not board location, speeded responses to targets near the hand. This pattern of results replicated previous studies using covert orienting paradigms with highly predictive cues. In Experiment 2, we used the same basic paradigm but emphasized the location of the response hand. Results now showed speeded responses to targets near response locations. Together these experiments demonstrated that top-down instructional sets (i.e., what is considered to be most relevant to task performance) can change the processing priority of hand location by influencing the strength of top-down, as compared with bottom-up, inputs competing for attention resources.
\end{abstract}

Keywords Spatial attention - Cue predictability · Embodiment · Top-down · Task relevance $\cdot$ Multisensory integration

\footnotetext{
M. J. Strom • C. L. Reed ( $\bowtie)$

Department of Psychology, Claremont McKenna College, 850 Columbia Ave, Claremont, CA 91711, USA

e-mail: clreed@cmc.edu

J. P. Garza • R. J. Roberts Jr. • C. L. Reed

Department of Psychology, University of Denver,

2155 S. Race St., Denver, CO 80208, USA

C. E. Wright

Department of Cognitive Sciences, 2201 Social \& Behavioral Sciences Gateway Building (SBSG), University of California Irvine, Irvine, CA 92697-5100, USA
}

Recent spatial attention studies have demonstrated attentional biases for visual stimuli presented near the hand (Reed, Betz, Garza, \& Roberts, 2010; Reed, Grubb, \& Steele, 2006). In experiments using covert orienting paradigms with predictive cues, speeded response times are observed for targets appearing near the hand, rather than for targets on the opposite side of the display. These results suggest the additional contribution of visual-tactile bimodal processes to visuospatial processing for the region of space near the hand, as well as proprioceptive inputs indicating hand location. Furthermore, the topography of the attentional bias near the hand appears to be related to functional capabilities of the hand. The bias is strongest for targets appearing in grasping space next to the palm, as compared with equidistant locations behind the hand or near the forearm. Together, these studies suggest that the ability of the hand to perform actions on nearby objects affects the distribution of spatial attention.

An open question is whether the hand's biasing of attention is affected not only by additional perceptual contributions, but also by task expectations. The selection of contending objects by visual attention is a competitive process among sensory-related bottom-up inputs and top-down sources of control to direct attention to particular objects or spatial locations (Kastner \& Ungerleider, 2000). Bottom-up neural mechanisms are largely automatic and select on the basis of stimulus features (e.g., luminosity). Multimodal perceptual inputs from visual, proprioceptive, and tactile systems about hand position may act together to increase their salience in the selection process. Top-down mechanisms bias this selection to objects relevant to current behavior and goals. The placement of the hand can bias goaldirected behavior because its location and grasping function suggests that a stimulus near it may be important for upcoming action. Thus, a hand positioned in a particular location in the visual field is likely to influence attention from both bottom-up and top-down directions. Although recent work has argued that the effect of the body on visual 
processing may be attributed to early, sensory-related processing (e.g., bimodal neurons, figure-ground segregation; Cosman \& Vecera, 2010; Reed et al., 2010; Reed et al., 2006), little work has investigated how top-down influences and task expectations interact with the hand's effect on visual attention.

We investigate how top-down influences, such as cue predictability and instructional set, might alter the hand's influence on spatial attention. In many studies that investigate the hand's influence on spatial attention, the focus of the task is on a hand's location relative to the target or the set of targets (Tseng \& Bridgeman, 2011). In some studies, the hand hypothesized to affect visual attention is distinct from the responding hand and is otherwise irrelevant to completing the task (Cosman \& Vecera, 2010; Reed et al., 2010; Reed et al., 2006), while in other studies the responding hand may be positioned near the target (Abrams, Davoli, Du, Knapp, \& Paull, 2008; Tseng \& Bridgeman, 2011). In all such experiments, however, participants are aware of their hand position near the monitor screen, whether its location is relevant to task performance or not. Its position must be part of the general task instructions because it is not a typical posture for working at a desktop computer. This awareness potentially contributes to top-down sources of bias directly related to the hand, especially in cases where hand position is part of task performance, thereby engaging higher level cognition. Indeed, placing a different emphasis on hand position when it is relevant versus irrelevant to the task may affect the nature of attentional hand bias, and differential effects of the hand on visual processing and attention may be related to the perceived focus or goal of the task.

Here, we investigated how hand position affects visual processing and the extent to which hand bias is obligatory by changing the priority of the hand's functions. Specifically, we manipulated the instructional set to change the task focus from the hand's location relative to the target to the hand's location for the response. Experiment 1 emphasized the hand's position relative to target location. Experiment 2 changed the emphasis to response location. Together, they demonstrated how top-down influences can mediate the body's contributions to spatial attention.

\section{Experiment 1}

We compared hand and visual anchor conditions and emphasized the location of the hand or the visual anchor relative to potential target locations (Reed et al., 2006, Experiment 2). Because Reed et al. (2006; Reed et al., 2010) utilized predictive visual cues, top-down expectations for target locations may have interacted with bias from the hand. Hand bias did not interact with visual cue validity effects in these studies, potentially because the visual cuing was reinforced to an extent that obscured the detection of any interactions. In order to rule out interactive effects from other potential top-down sources of bias, it was important first to establish that the same types of hand bias effects would be observed when cues were not predictive.

\section{Method}

\section{Participants}

Thirty-four right-handed undergraduates participated for extra credit.

\section{Stimuli}

Stimuli were constructed using Microsoft PowerPoint and presented on a 17-in. monitor using E-Prime 1.0 software (Psychological Software Tools, Pittsburgh, PA). Stimuli were black on a white background: A fixation cross $\left(2^{\circ}\right)$ was flanked by cue squares $10 \mathrm{~cm}$ from the fixation on either side; a target dot $\left(1.8^{\circ}\right)$ appeared in one of the two squares. The visual anchor was a $2 \times 4$ in. board sharing similar dimensions as the hand and arm. Participants' arms and the board were held next to target box locations on their sides and were supported by a brace (Reed et al., 2006, Experiment 2).

\section{Procedure}

Participants performed a covert attention task with nonpredictive cues (e.g., Posner, Inhoff, Friedrich, \& Cohen, 1987). Participants were instructed to fixate on the cross throughout the experiment. At the start of each trial, two black boxes and a central cross were presented for 1,500-3,000 ms. One box's border darkened as a cue, followed $200 \mathrm{~ms}$ later by the appearance of a target in one of the boxes. Participants responded to the target appearance by clicking on a one-button mouse. Targets appeared either in the cued box on valid trials ( $45 \%$ of total) or in the opposite, noncued box on invalid trials (45\%). During catch trials (10\%), no target appeared in either location, and participants were required to withhold a response. The target disappeared upon response. Participants were told that cues did not predict target location.

Before each block of trials, participants viewed a text screen indicating the hand placement condition ("hand" or "block") and side ("left" or "right"). For the hand conditions, participants were told to place their hand next to the outside of the indicated target box. Their arm was supported on a platform, and their palm faced the box in a loose grasp posture (Fig. 1a). For the block conditions, participants were told to place their hand in their lap and the experimenter 

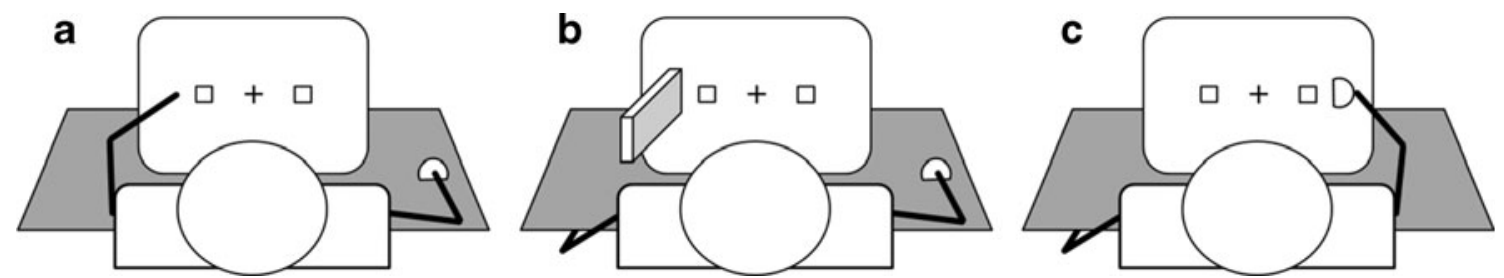

Fig. 1 Schematic depiction of three basic conditions across both experiments. a Hand condition of Experiment 1 and desk condition of Experiment 2: One hand is held up near a target location, and the response is made on a mouse with the other hand on the desk. b Board

placed the board on the platform and positioned its side next to the outside of the target box. Responses to the target were made with the index finger of the opposite hand on a desktop mouse located beneath the opposite target box. After 20 practice trials, eight blocks were presented in random order, with each condition/side combination occurring twice. Each block consisted of 50 trials, for a total of 400 trials. The experiment lasted approximately $45 \mathrm{~min}$.

\section{Results and discussion}

Mean response times (RTs) were calculated for each condition. Following Reed et al. (2006; Reed et al., 2010), trials outside a time window from 200 to $900 \mathrm{~ms}$ after the target onset were excluded to eliminate anticipation and inattention errors (fewer than $1 \%$ of trials).

A repeated measures 2 (condition: hand, board) $\times 2$ (anchor side: left, right) $\times 2$ (target side: left, right $) \times 2$ (validity: valid, invalid) ANOVA was conducted. Valid trials (395.66 ms [8.50]) were responded to more quickly than invalid trials (405.14 ms [11.60]) [validity: $F(1,33)=4.27$, $M S E=2,858.83, p=.047]$. Importantly, performance was moderated by hand position, but not board position, relative to the target. Targets appearing next to the hand were detected relatively more quickly than targets appearing away from the hand; there was little difference between which side the target appeared on relative to board position [Fig. 2; condition $\times$ anchor side $\times$ target side interaction: $F(1,33)=10.63, M S E=324.82, p=.003]$. Post hoc comparisons indicated that the anchor side $\times$ target side interaction occurred only for hand conditions, $F(1,33)=$ 29.80, MSE $=332.63, p<.0001$, and not board conditions, $F(1,33)<1, M S E=444.49$, n.s.]. However, this hand position bias occurred for both validly and invalidly cued trials: The four-way interaction that included cue validity was not significant, $F(1,33)=1.15, M S E=234.26, p=.29$. No other main effects or interactions approached significance $(p>.121)$.

In sum, when target location could not be predicted, hand location did not interact with cue validity. Attention was biased specifically for targets appearing near the hand, but not for targets appearing near nonbody anchors. Thus, the condition of Experiment 1: A board is positioned near the target location in place of the hand as a visual anchor. $\mathbf{c}$ Screen condition of Experiment 2: The responding hand is positioned near the target location, and the nonresponding hand is placed on the lap

hand bias occurs even when attention is cued exogenously and does not differ from that found when cues are highly predictable.

\section{Experiment 2}

The purpose of Experiment 2 was to determine whether task context could affect the hand's ability to bias attention. Using the same basic paradigm as that in Experiment 1, we changed the instructional set to emphasize the location of the hand response, rather than the location of the nonresponding hand, relative to the target. One hand was always next to target locations on the screen, but responses were made either on the desk or on the screen next to the target. Critically, the instructions preceding each block specified the response hand position (e.g., "Screen Left"). If top-down changes to task relevance can overcome bottom-up influences of hand location, the RT bias should shift from hand location to response location.

\section{Method}

\section{Participants}

Thirty-four right-handed undergraduates participated for credit in psychology courses.

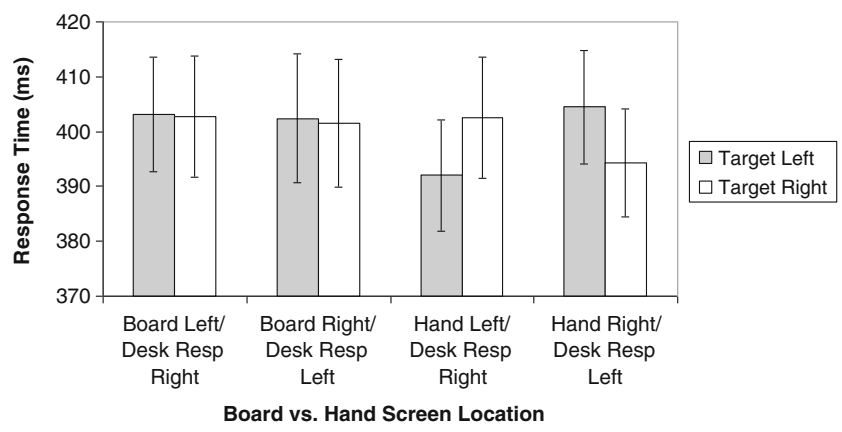

Fig. 2 Experiment 1: Board versus hand screen location. Mean response times for board or hand location side and target side. Error bars indicate standard errors 


\section{Stimuli and procedure}

The same stimuli and experimental paradigm were used as in Experiment 1. The procedure was similar to Experiment 1 , except that the focus was changed to the location of the response. Before each block of trials, participants viewed a text screen indicating the response location condition ("screen" or "desk") and side ("left" or "right"). For the screen response conditions, a wireless mouse was attached to a flat, vertical bookend fixed to the platform support so that it could be positioned adjacent to the indicated box on the screen. With the arm supported by the platform, participants were instructed to loosely grasp the mouse with their hand so that the palm (and the flat side of the mouse) faced the box and to press the mouse button with that index finger (Fig. 1c). For the desk response conditions, participants were told to respond on the mouse located on the desk underneath the indicated box using the index finger of the same side hand; they placed the opposite hand next to the other target box so that their palm faced the box and their arm was supported by the platform (Fig. 1a).

\section{Results and discussion}

Mean RTs were calculated as in Experiment 1. A repeated measures 2 (response location: screen, desk) $\times 2$ (response side: left, right) $\times 2$ (target side: left, right) $\times 2$ (validity: invalid, valid) ANOVA was conducted (Fig. 3). Participants responded more quickly to valid (339.36 $\mathrm{ms}$ [7.40]) than to invalid (349.51 ms [8.88]) cues [validity effect: $F(1,33)=$ $12.95, M S E=1,080.50, p=.001]$. No other main effects were significant (all $p \mathrm{~s}>.146$ ). The response side $\times$ target side interaction was significant, $F(1,33)=5.21, M S E=$ 377.08, $p=.029$, indicating shorter RTs for targets appearing on the same side as the response hand. No other interactions were statistically significant (all $p \mathrm{~s}>.224$ ). Specifically, neither the response location $\times$ response side $\times$ target side interaction, $F(1,33)<1, M S E=261.06$, n.s.,

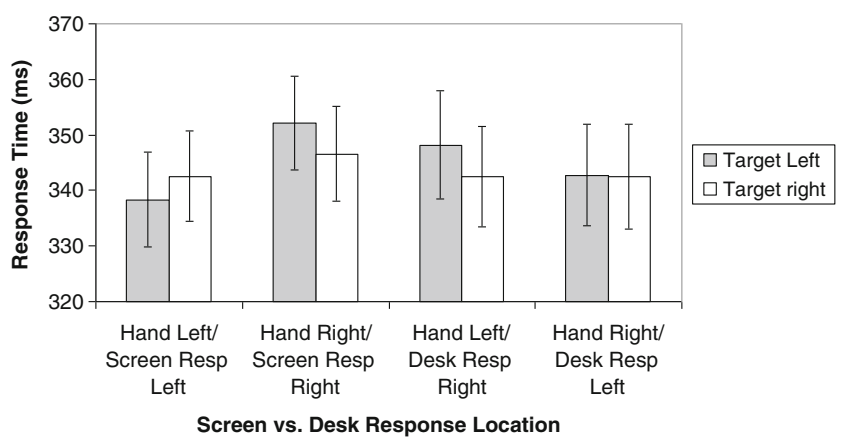

Fig. 3 Experiment 2: Screen versus desk response location. Mean response times for screen or desk response location and target side. Error bars indicate standard errors nor the four-way interaction, $F(1,33)<1, M S E=477.76$, n.s., was significant.

Because previous studies indicated that hand proximity to the target influences the strength of the hand bias, we conducted separate comparisons for the response side $x$ target side interactions for the screen and desk conditions to determine whether the response effect was greater for the response near the target than for the response further from the target. A significant effect was found for the screen condition, $F(1,33)=4.77, M S E=338.53, p=.036$, but not for the desk condition, $F(1,33)=1.70, M S E=299.60$, $p=.202$. Thus, the bias was statistically reliable for the screen response condition, but not for the desk response condition, in this experiment. However, although not statistically significant, the pattern of means in the desk response condition was in the opposite direction from that in the hand condition in Experiment 1, which was a physically identical condition. This lack of the hand bias effect in Experiment 2 suggested that the top-down prioritization for response location modified attention orienting.

To confirm that task focus changed attentional bias, we conducted a post hoc comparison between Experiments 1 and 2 for identical physical conditions with differing instructional sets (i.e., Experiment 1's hand condition was physically identical to Experiment 2's desk condition). Although the between-subjects design necessary to compare across the two experiments potentially limits statistical pow$\mathrm{er}$, it also guaranteed that each individual was presented with only a single instruction set, minimizing potential crossinstruction effects. Accordingly, a 2 (experiment) $\times 2$ (hand side $) \times 2$ (target side) ANOVA was conducted. The significant three-way interaction, $F(1,66)=18.34, M S E=$ $530.65, p<.0071$, indicated that the direction of the bias was changed merely by changing the relative importance of response location over hand location. Thus, top-down effects of task relevance can change attention bias from hand location to response location in otherwise identical conditions.

\section{General discussion}

This study demonstrated that body-based attention bias could be manipulated by top-down influences such as task relevance or instruction set. Using covert orienting tasks with lateralized, nonpredictive cues, it showed that attention bias is specific to the hand but that the hand's influence on processing can be mediated by task instructions regarding what is important to performance. Experiment 1 emphasized the location of the hand irrelevant to task performance and confirmed hand-specific effects of attentional bias. When a hand was placed next to a potential target location, responses were faster to targets appearing near the hand than 
to those appearing away from the hand. This effect did not occur for nonhand visual anchors placed next to target locations. These results are consistent with those found in Reed et al. (2006) for highly predictable cues but were replicated here with nonpredictive cues, indicating that the influence of the hand is not dependent on cue predictability. The present results support the conclusion that expectation of cue location does not affect bottom-up sensory inputs for attentional competition that could potentially obscure interactions between hand location and cue location (Abrams et al., 2008) or any independent effects of endogenous (voluntary) and exogenous (automatic) orienting (Klein \& Shore, 2000).

In contrast, emphasizing different aspects of the task can influence the hand's bias on spatial attention. Experiment 2 changed the task emphasis to response location, thereby changing the bias from irrelevant hand location to functional hand response location: Speeded responses were observed for targets appearing on the side of the response hand. This bias was stronger when the response hand was held near the target than when positioned on the desk. That hand proximity to the target produces a strong effecter than just response location alone suggests that bottom-up sensory inputs also contribute to performance. Finally, post hoc comparisons of results from physically identical conditions from the two experiments confirmed the shift in bias produced by the instructional set. Thus, hand effects are not just caused by an awareness of hand position.

The top-down mediation of hand-specific location effects on attention observed here is consistent with the biased competition model of attention (Desimone \& Duncan, 1995; Duncan, 2006). In this model, competition for representation of various sensory inputs occurs in the visual cortex on the basis of the featural properties of the stimuli but is biased in a top-down direction from more anterior neural regions with information about current goals and behavioral control. Thus, changes in instructional set can affect attention to different possibilities for action within a task by influencing the topdown bias. Specifically, visual and proprioceptive inputs from the hand may interact with competing visual stimuli to direct attention, but the task demand of holding the hand in a specific position may additionally bias the competition for the side of space in which the hand is located. Furthermore, instruction set and experimental context (i.e., the goals of all of the tasks across condition blocks in a particular experiment, and not just the current task goal within a particular block) can shape this top-down bias and, ultimately, influence attention allocation to be suitable for the most relevant of the upcoming actions. In this study, bottom-up hand location inputs contributed to the overall response in a dynamic competition between task relevance and perceptual saliency. However, the same condition (i.e., one hand near the target and the contralateral hand responding on a desk) produced two opposing data patterns merely by emphasizing the response location over the hand location.

Future studies investigating the hand bias effect on attention should continue to identify how bottom-up and topdown inputs differentially contribute to the overall outcome for attention allocation in the presence of the individual's hand. Although the effect of instruction set occurred across the two experiments in a between-subjects design in this study, future study designs might be able to manipulate task instruction in such a way that one instruction set minimally affects ongoing processing in later conditions in which the instruction set has changed. More generally, this study highlights the importance of task instruction in cognitive experimental design and suggests that it would be a good potential candidate for further study as a concrete source of top-down attentional bias.

Acknowledgements The authors would like to thank Casey Wieland for his help in running participants in this experiment. This work was supported in part by a Partners-In-Scholarship grant from the University of Denver.

\section{References}

Abrams, R. A., Davoli, C. C., Du, F., Knapp, W. H., \& Paull, D. (2008). Altered vision near the hands. Cognition, 107, 10351047.

Cosman, J. D., \& Vecera, S. P. (2010). Attention affects visual perceptual processing near the hand. Psychological Science, 21, 1254-1258.

Desimone, R., \& Duncan, J. (1995). Neural mechanisms of selective visual attention. Annual Review of Neuroscience, 18, 193-222.

Duncan, J. (2006). EPS Mid-Career Award 2004: Brain mechanisms of attention. Quarterly Journal of Experimental Psychology, 59, 2-27.

Kastner, S., \& Ungerleider, L. (2000). Mechanisms of visual attention in the human cortex. Annual Review of Neuroscience, 23, 315-341.

Klein, R. M., \& Shore, D. I. (2000). Relations among modes of visual orienting. In S. Monsell \& J. Driver (Eds.), Attention and performance XVIII Control of cognitive processes (pp. 195-208). Cambridge, MA: MIT Press.

Posner, M. I., Inhoff, A., Friedrich, F. J., \& Cohen, A. (1987). Isolating attentional systems: A cognitive-anatomical analysis. Psychobiology, $15,107-121$.

Reed, C. L., Betz, R., Garza, J. P., \& Roberts, R. J., Jr. (2010). Grab it! Biased attention in functional hand and tool space. Attention, Perception, \& Psychophysics, 72, 236-245.

Reed, C. L., Grubb, J. D., \& Steele, C. (2006). Grasping attention: Behavioral consequences of bimodal neurons. Journal of Experimental Psychology. Human Perception and Performance, 32, 166-177.

Tseng, P., \& Bridgeman, B. (2011). Improved change detection with nearby hands. Experimental Brain Research, 209, 257-269. 